\title{
Spin-Polarized Electron Scattering at Single Oxygen Adsorbates on a Magnetic Surface
}

\author{
K. von Bergmann, ${ }^{1, *}$ M. Bode, ${ }^{1}$ A. Kubetzka,,${ }^{1}$ M. Heide, ${ }^{2}$ S. Blügel, ${ }^{2}$ and R. Wiesendanger ${ }^{1}$ \\ ${ }^{1}$ Institute of Applied Physics and Microstructure Research Center, University of Hamburg, \\ Jungiusstrasse 11, 20355 Hamburg, Germany \\ ${ }^{2}$ Institut für Festkörperforschung, Forschungszentrum Jülich, 52425 Jülich, Germany
}

(Received 2 July 2003; published 27 January 2004)

\begin{abstract}
Scanning tunneling spectroscopy (STS) on the system of isolated oxygen atoms adsorbed on the double layer of $\mathrm{Fe}$ on W(110) shows highly anisotropic spatial oscillations in the local density of states in the vicinity of the adsorbates. We explain this in terms of a single-particle model as electron waves being scattered by the potential induced by the presence of the oxygen atoms. Analysis of the wavelength of the standing electron waves and comparison with ab initio spin-resolved electronic structure calculations reveal that minority-spin bands of $d$-like symmetry are involved in the scattering process. By applying spin-polarized STS, we observe this standing wave pattern on one particular type of magnetic domain of $\mathrm{Fe}$ on $\mathrm{W}(110)$ only, thereby proving that the standing electron waves are highly spin polarized.
\end{abstract}

DOI: 10.1103/PhysRevLett.92.046801

Scanning tunneling microscopy (STM) and spectroscopy (STS) are powerful tools to study the interplay between structural and electronic properties of surfaces and to reveal the orbital symmetry of electronic states. A deeper understanding of magnetic properties, though, requires the use of spin-polarized STM-STS investigations which yield further information about the spin character of the electronic states involved. By making use of the high spatial and energy resolution of STM and STS, modulations of the local electron density of states on the nanometer scale as caused by scattering at impurities or step edges have been studied intensely in recent years. The analysis of electron standing wave patterns [1-9] allowed the acquisition of characteristic properties as the symmetry of the wave function, the dispersion relation, or the lifetime of the electronic states. All previous experiments were performed with nonmagnetic tips resulting in spin-averaged data, although scattering processes at magnetic impurities or at nonmagnetic impurities on magnetic surfaces should result in spin-polarized (SP) electron waves. These are of fundamental importance for the understanding of single-particle as well as many-body processes, e.g., the RKKY interaction or the interaction between Kondo impurities.

A well studied magnetic model system is Fe on W(110) which has been investigated previously by means of spinintegrated and spin-polarized STM-STS measurements. Together with $a b$ initio electronic structure calculations this led to a comprehensive understanding of its electronic and magnetic properties [10,11].

In this Letter we report on the observation of spinpolarized spatial variations of the local density of states (LDOS) in the vicinity of single oxygen impurities adsorbed on the ferromagnetic Fe double layer on W(110) by SP-STS. The results are compared to first-principles spin-resolved electronic structure calculations. We show
PACS numbers: 73.20.-r, 68.37.Ef, 71.15.Mb, 75.70.-i

that the observed LDOS oscillations can be assigned to minority-spin bands of $d$-like character. Spin-polarized STS measurements show that they can be observed on one particular type of magnetic domain only, confirming the unique ability of SP-STS to investigate the spin character of electronic states together with the orbital symmetry at high spatial resolution.

The experiments have been performed in a cryogenic STM [12] attached to a five-chamber ultrahigh vacuum system. During measurements tip and sample are held at $T=13 \pm 1 \mathrm{~K}$. Differential conductance $(d I / d U)$ spectra are acquired with an open feedback loop at the given stabilization parameters, while $d I / d U$ maps are measured with closed loop at the indicated sample bias voltage $U$. We used clean and $\mathrm{Gd} / \mathrm{Fe}$-coated $\mathrm{W}$ tips for spin-integrated and out-of-plane sensitive magnetic measurements, respectively. Fe was evaporated from a rod heated by electron bombardment ( $p \leq 1 \times 10^{-10} \mathrm{mbar}$ ). To achieve step-flow growth, the $\mathrm{W}(110)$ substrate was held at $T=500 \mathrm{~K}$ during thin film deposition.

Upon Fe deposition the sample surface was exposed to about $0.01 \%$ of a Langmuir of $\mathrm{O}_{2}$ at room temperature leading to dissociative chemisorption of $\mathrm{O}$ atoms in the twofold long-bridge site [13]. As can be seen in the constant-current topography of Fig. 1(a) and the corresponding $d I / d U$ map in 1(b) they are distributed randomly and imaged as depressions at $U=+0.7 \mathrm{~V}$. The local tunneling spectrum of Fig. 1(c) reveals that for $U \leq$ $+0.8 \mathrm{~V}$ oxygen locally causes an attenuation of the $d I / d U$ signal compared to clean Fe spectra.

Figure 1(d) shows high-resolution $d I / d U$ maps around a single oxygen atom at $U=+0.3 \mathrm{~V}$ and $U=-0.1 \mathrm{~V}$ as indicated with highly anisotropic patterns. The black lines in Fig. 1(d) indicate the positions of the line profiles which are plotted in Fig. 1(e). On the left side of Fig. 1(d) we observe oscillations in the $d I / d U$ map along the [001] 


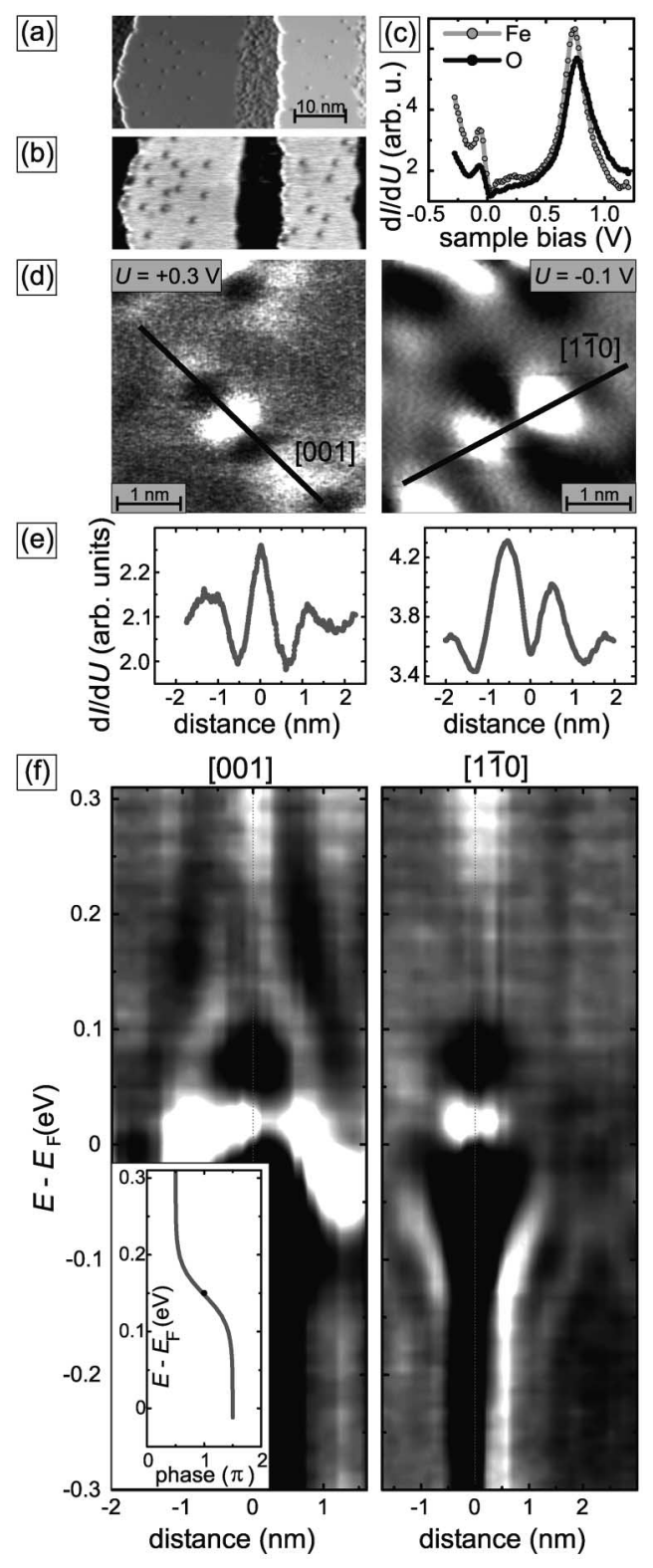

FIG. 1. STM-STS measurements of Fe on W(110) after dosing $\approx 0.01 \% \mathrm{~L}$ of $\mathrm{O}_{2}$ : (a) Topography: $\mathrm{O}$ on $1.8 \mathrm{ML} \mathrm{Fe} / \mathrm{W}(110)$; (b) $d I / d U$ map corresponding to (a) at $U=+0.7 \mathrm{~V}$; (c) $d I / d U$ spectra on the double layer of $\mathrm{Fe} / \mathrm{W}(110)$ and on top of an oxygen atom; the stabilization parameters are $U=1.2 \mathrm{~V}$ and $I=0.5 \mathrm{nA}$; (d) $d I / d U$ maps of single oxygen adsorbates, sample bias voltage $U$ as labeled; the black lines indicate the positions of the corresponding line profiles shown in (e); (f) data from $d I / d U$ spectroscopy (stabilization parameters: $U=+0.3 \mathrm{~V}, I=0.25 \mathrm{nA}$; logarithmic gray scale): energydependent $d I / d U$ line profiles across a single oxygen adsorbate along the two crystallographic axes; inset: energy-dependent phase relation derived from the data.

direction of the substrate with a maximum of the $d I / d U$ signal at the center of the oxygen atom (distance $d=$ $0 \mathrm{~nm}$ ). On the right side of Fig. 1(d) the $d I / d U$ map measured at $U=-0.1 \mathrm{~V}$ shows oscillations along the
[110] direction of the substrate. At this particular voltage we observe a local minimum at $d=0 \mathrm{~nm}$. The oscillations of the $d I / d U$ signal show a strong attenuation with increasing distance from the center of the oxygen adsorbate.

We interpret the oscillations in the differential conductance in terms of standing electron waves and explain them within a single-particle model. In the proximity of impurities, electron waves are scattered by the impurity potential. Coherent interference of the incoming with the reflected electron wave results in spatial oscillations of the density of states. The observed real-space oscillations can be analyzed regarding their periodicity and phase. The periodicity contains information about the wave vector component $k_{\|}$of the electron wave. To further investigate the scattering behavior at the oxygen atoms we probed the electronic structure in the vicinity of the adsorbates at different energies.

Figure 1(f) shows data from $d I / d U$ spectroscopy. The gray scale represents the $d I / d U$ signal as a function of distance from the oxygen adsorbate divided by the $d I / d U$ signal of the clean Fe surface at the corresponding energy. In the context of this Letter we are interested in the long-range standing wave pattern which is due to the scattering process. Therefore, we do not consider the local density of states at the position of the oxygen atom $(d=$ $0 \mathrm{~nm}$ ) because we expect to see a rather large impact of a topographic effect, the correction of which may not be straightforward. Instead, the area of interest for this analysis is further away from the oxygen atom $(d>$ $0.5 \mathrm{~nm}$ ) where the corresponding constant-current image at the stabilization parameters is flat. Here, the undisturbed anisotropic standing wave patterns on the $\mathrm{Fe}$ surface are observed, and no additional corrections are necessary before analyzing the standing wave patterns [2]. Along the [001] direction we can recognize a characteristic pattern in the $d I / d U$ intensity above the Fermi level $E_{\mathrm{F}}$. The course of maxima and minima is clearly visible. A closer inspection reveals an energy-dependent wavelength of these oscillations. Furthermore, the phase of the standing wave pattern due to the scattering process of electron waves at the oxygen atom can be determined from the distance between the first maximum of the standing wave pattern and the position of the point scatterer in Fig. 1(f). This distance in relation to the wavelength gives the phase where one wavelength corresponds to $2 \pi$. For example, at $E_{\mathrm{F}}$ we observe a phase shift of about $-\frac{\pi}{2}$ for the scattering process. We identify this as an energy-independent background phase as also assumed for other scatterers [6,9]. The inset of Fig. 1(f) shows that a phase shift of $\pi$ is observed for the [001] direction at $E \sim$ $+0.15 \mathrm{eV}$. The [1110] direction shows a characteristic pattern of the density of states below $E_{\mathrm{F}}$ with a similar phase relation as for [001] and an inflection point at $E \approx$ $-0.08 \mathrm{eV}$ (not shown). Both energy-dependent phase shifts could be explained by resonances. 


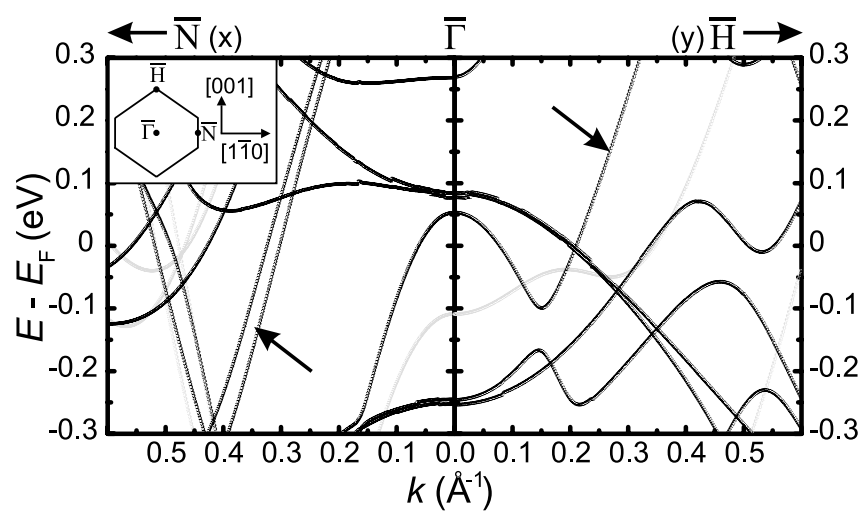

FIG. 2. Spin-resolved band structure calculation for $2 \mathrm{ML}$ Fe/W(110); black: minority bands; gray: majority bands; inset: 1. Brillouin zone for a (110) surface of a bcc crystal and crystallographic axes in real space.

We have compared the experimental results with firstprinciples electronic structure calculations based on density functional theory. Figure 2 shows the calculated spin-resolved band structure of $2 \mathrm{ML} \mathrm{Fe}$ adsorbed pseudomorphically on either side of a five layer W(110) slab. Minority and majority bands are plotted as black and gray lines, respectively. From our data we extracted the period of the standing waves at various energies and calculated the $k$ vectors of the corresponding electron wave functions. This allows the comparison between experimental and theoretical data. The oscillations in [001] can be assigned to a rising minority-spin band in the $\overline{\Gamma H}$ direction indicated by the arrow in Fig. 2. An analysis of the eigenstate reveals that this band consists basically of a superposition of $d_{z^{2}}$ and $d_{z y}$-like states in the Fe surface layer. Localized in the vacuum is $10 \%$ of this state, measured always from a position of $1.2 \AA$ above the surface Fe layer. The oscillations in [11̄0] result from scattering involving one of the two bands which are indicated by the arrow in the $\overline{\Gamma N}$ direction. Both are of minority-spin character. The band closer to $\bar{\Gamma}$ is a $d_{z x}$-like state in the Fe surface layer with $8 \%$ of its weight localized in the vacuum. The other one is a superposition of $d_{z^{2}}, d_{z x^{-}}$, and $d_{x^{2}-y^{2}}$-like states in the Fe surface layer and only $1 \%$ is localized in the vacuum. For this reason we assume that the band visible in the scattering process is the one closer to $\bar{\Gamma}$. As we show below, these two bands allow a precise modeling of the observed course of maxima and minima in the $d I / d U$ signal.

If the above-mentioned interpretation is correct, we expect to see a significant spin dependence in the electron scattering by spin-polarized STS. In particular, SP-STS measurements on different types of magnetic domains with the same magnetic tip should provide information about the spin characteristics of the electronic states involved in the scattering process. The well-known magnetic structure of $2 \mathrm{ML} \mathrm{Fe} / \mathrm{W}(110)$, which is characterized by about $20-25 \mathrm{~nm}$ wide, perpendicularly mag- netized domains [11], was found to be unchanged by the oxygen adsorbates. Full $d I / d U$ spectroscopy was performed around a single oxygen atom adsorbed on one particular type of domain (A). Afterwards, the same measurement was performed around another oxygen atom on the oppositely magnetized domain B. Eventually, the measurement was repeated on the initial domain A to exclude any unwanted tip change.

The experimental data are presented in Fig. 3. Indeed, the $d I / d U$ map in Fig. 3(a) demonstrates that the characteristic patterns in the differential conductance as known from spin-averaged measurements [cf. Fig. 1(e)] can be recognized on domain $\mathrm{A}$, while they are nearly absent on domain B. Figure 3(b) shows high-resolution $d I / d U$ maps at the labeled voltages $U$. Lines indicate the position of the corresponding line profiles shown in 3(c). Around the oxygen atom on domain A the above-mentioned scattering states are enhanced for both high-symmetry directions, whereas on domain $\mathrm{B}$ they can hardly be detected. The $d I / d U$ variation of these line profiles was analyzed quantitatively by measuring the difference of the first maximum and minimum adjacent to the position of the oxygen atom [cf. Fig. 3(c)] and then dividing it by their mean value. Assuming a reading error of 0.025 arbitrary units we calculate the following ratios: [001]: domain $\mathrm{A}, 13.5 \pm 3.4 \%$, and domain $\mathrm{B}, 3.1 \pm 2.8 \%$; [11̄0]: domain A, $14.1 \pm 0.6 \%$, and domain $\mathrm{B}, 4.4 \pm$ $0.5 \%$. The factor of about 3-4 between domains $A$ and $\mathrm{B}$ proves the strong spin polarization of the electron waves around the impurity.

We have compared the calculated band structure of Fig. 2 with the SP-STS measurements by converting the calculated dispersion relation $E(\vec{k})$ of the bands identified to be involved in the scattering process into an energydependent electron wavelength $\lambda(E)=2 \pi / k(E)$. Then $\lambda / 2$ is the periodicity of the LDOS, which is proportional to the STS signal. After correction with the experimentally determined energy-dependent phase [cf. inset of Fig. 1(f)], the positions of the maxima and minima of the density of states can be simulated as a function of energy and of distance from the center of the oxygen atom. The result is shown in Fig. 3(d) together with data from $d I / d U$ spectroscopy for both domains and both high-symmetry directions. Even though the band structure calculation does not account for adsorbate-induced modifications of the electronic structure but only models the oxygen atom as a point scatterer, the simulation is in excellent agreement with the experimental data.

In summary, we observed highly anisotropic scattering states around individual oxygen atoms on the double layer of Fe on W(110). Comparison of the experiment with firstprinciples spin-resolved electronic structure calculations reveals that the electronic states involved in the scattering process are of minority-spin character. STS measurements with a spin-polarized tip support this assumption: we observe oscillations in the density of states on one 


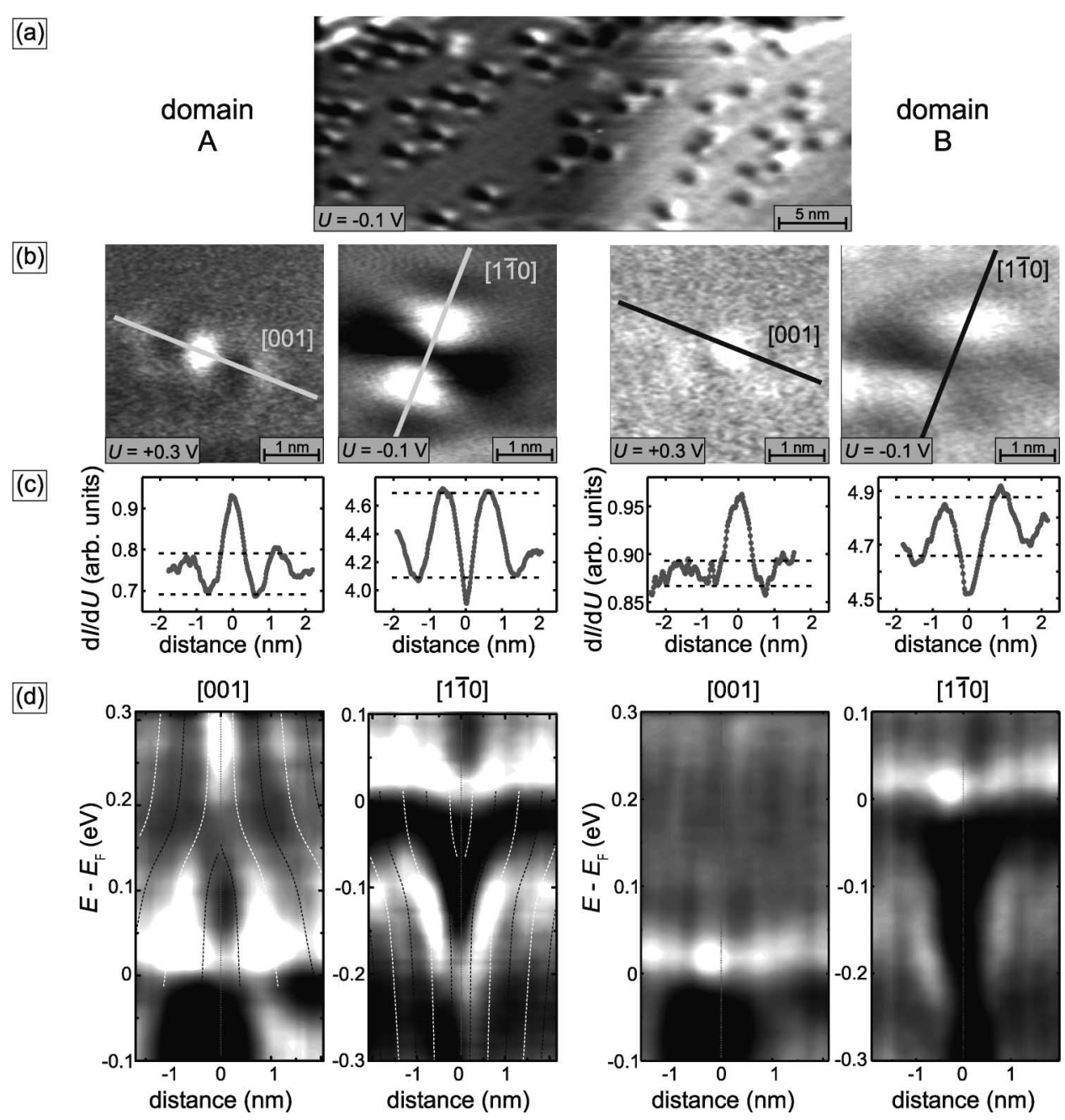

FIG. 3. Spin-polarized STS measurements (with a Gd/Fe coated W-tip): (a) $d I / d U$ map showing oxygen adsorbates on two different magnetic domains, $\mathrm{A}$ and $\mathrm{B}$; (b) $d I / d U$ maps of single oxygen adsorbates; the lines indicate the positions of the corresponding line profiles shown in (c); (d) data from $d I / d U$ spectroscopy (stabilization parameters: $U=+0.3 \mathrm{~V}, I=0.25 \mathrm{nA}$; logarithmic gray scale) on both domains along the two crystallographic axes; the black and white dotted lines in the graph for domain A indicate the minima and maxima simulated on the basis of the band structure calculation and the experimentally determined phase relation. Note the different energy range for the [001] and [110] directions.

type of domain only, which proves that they are, indeed, highly spin polarized. We expect that this method can be applied to a great variety of systems involving spinpolarized electron states at surfaces.

We acknowledge O. Pietzsch for technical assistance and financial support from the DFG.

*Electronic mail: kbergman@physnet.uni-hamburg.de

[1] M. F. Crommie, C. P. Lutz, and D. M. Eigler, Nature (London) 363, 524 (1993).

[2] J. Li, W.-D. Schneider, and R. Berndt, Phys. Rev. B 56, 7656 (1997).

[3] J. Li et al., Phys. Rev. Lett. 81, 4464 (1998).
[4] O. Jeandupeux et al., Phys. Rev. B 59, 15926 (1999).

[5] B. G. Briner et al., Phys. Rev. B 58, 13931 (1998).

[6] G. A. Fiete et al., Phys. Rev. Lett. 86, 2392 (2001).

[7] J. I. Pascual et al., Phys. Rev. B 63, 241103 (2001).

[8] O. Y. Kolesnychenko et al., Nature (London) 415, 507 (2002).

[9] M. A. Schneider, L. Vitali, N. Knorr, and K. Kern, Phys. Rev. B 65, 121406 (2002).

[10] M. Bode et al., Phys. Rev. Lett. 89, 237205 (2002).

[11] A. Kubetzka, M. Bode, O. Pietzsch, and R. Wiesendanger, Phys. Rev. Lett. 88, 057201 (2002).

[12] O. Pietzsch et al., Rev. Sci. Instrum. 71, 424 (2000).

[13] W. Erley and H. Ibach, Solid State Commun. 37, 937 (1981). 